\title{
Orientação sexual e fatores associados em homens homossexuais
}

\author{
Sexual orientation and associated factors in homosexual men
}

\author{
Fabiano Augusto Teixeira, ${ }^{1}$ Fernando Luiz Cardoso' \\ 'Universidade do Estado de Santa Catarina. Florianópolis, Santa Catarina, SC, Brasil.
}

Recebido em: 14/09/2016 / Aceito em: 17/02/2017 / Publicado em: 31/03/2017

fb_teixeira@hotmail.com

\section{RESUMO}

Esta pesquisa objetivou estimar a proporção de homens homossexuais e identificar os fatores associados à orientação sexual homossexual. Participaram 571 homossexuais e 75 bissexuais. Objetivo: estimar a proporção de homens homossexuais e identificar os fatores associados a orientação sexual homossexual. Material e Método: foi utilizado um questionário autoaplicado. Resultados: Encontrou-se associação em homens homossexuais e a orientação de gênero masculino $O R=$ $340,24(48,51$ - 2386,46), o grau de escolaridade $\mathrm{OR}=0,20(0,003-0,12)$ e a insatisfação com a imagem corporal por excesso $O R=0,124(0,49-0,314)$. Conclusão: conclui-se que os participantes homossexuais que mais se aproximam do número seis na Escala (gosta sexualmente de pessoas do mesmo sexo) são também que mais se percebem como masculino e que possui maior escolaridade.

Palavras-chave: Orientação sexual; Fatores associados; Homossexualidade; Gênero.

\section{ABSTRACT}

This study aimed to estimate the proportion of gay men and to identify factors associated with homosexual orientation. Sample was composed of 571 homosexuals and 75 bisexuals. Aim: estimate the proportion of gay men and to identify factors associated with homosexual orientation. Material and Method: we used a self-administered questionnaire. Results: an association was found in gay men and the male orientation $O R=$ 340.24 (48.51 to 2386.46), the level of education OR $=0.20(0.003$ to 0.12$)$ and dissatisfaction with body image by excess OR $=0.124$ (0.49 to 0.314). Conclusion: we conclude that homosexuals participants who come closest to the number six on the scale (sexually like people of the same sex) are also the most perceive themselves as men and have more schooling.

Keywords: Sexual orientation; Associated factors; Homosexuality; gender.

\section{INTRODUCÃ̃O}

Ao longo dos últimos dez anos, houve um aumento do número de pesquisas e publicações sobre o desenvolvimento da orientação sexual, ampliando o conhecimento dessas minorias sexuais. No entanto, conforme apontado, ${ }^{1}$ sabe-se muito pouco sobre a etiologia da orientação sexual, considerando que, há muitas suposições, mas poucos ainda são os dados ou evidências pautadas em uma criteriosa observação e análise comparativa.

E, pelo fato de ser objeto de estudo de diferentes áreas de conhecimento, como as ciências humanas e sociais, a produção científica não dialoga entre si, no entanto, já se sabe que a orientação sexual se desenvolve em fases; é multifatorial e seu conceito pode variar muito de área para área e de autor para autor. ${ }^{2}$

Embora diante desta falta de consenso na literatura nacional e internacional, este estudo adotará o entendimento trazido na literatura, ${ }^{3}$ o qual aponta que orientação sexual refere-se a relacionar-se no sentido do desejo sexual, tanto com pessoas do sexo oposto ou mesmo sexo ou até para ambos (homossexual, heterossexual e bissexual, respectivamente). Assim, refere-se à preferência sexual e física cuja compreensão impõe a atração sexual, comportamentos, fantasias e preferências emocionais ou sociais. ${ }^{4}$

Estudo $^{3}$ aponta a criação de uma escala bipolar que permite visualizar uma graduação contínua entre pessoas exclusivamente homossexuais ou heterossexuais. Essa criação ${ }^{5}$ considerou ambos os aspectos 
de orientação sexual: comportamento sexual (prática sexual) e fantasia sexual (orientação).

$\mathrm{Na}$ literatura, há evidências que homens homossexuais são os que associam, mais frequentemente, álcool com prática de sexo sem preservativo, ${ }^{2}$ maior prevalência de sífilis ${ }^{6}$ e alta proporção do uso irregular de preservativos. ${ }^{7}$ No entanto, são escassos os estudos que buscam investigar a proporção e os fatores associados à orientação sexual homossexual.

Assim, diante do exposto, este estudo objetiva es timar a proporção de homens homossexuais e identificar os fatores associados a orientação sexual homossexual.

\section{MATERIAL E MÉTODO}

Este estudo foi aprovado junto ao Comitê de Ética em Pesquisas com Seres Humanos da Universidade do Estado de Santa Catarina sob número 722.104.

Fizeram parte desta pesquisa 646 homens, todos maiores de 18 anos, frequentadores das praias de Florianópolis, Santa Catarina, Brasil. Destaca-se que a cidade que serviu para a coleta dos dados é uma região turística, cercada por 42 praias, sendo roteiro preferido do público constituído por Lésbicas, Gays, Bissexuais e Travestis, por ser considerada a $2^{\mathrm{a}}$ capital nacional do Brasil deste público. ${ }^{8}$

Os participantes foram abordados nas praias e receberam informações acerca dos objetivos da pesquisa. Feito isso, foram convidados a participar da pesquisa. Assim, os sujeitos que concordaram em fazer parte do estudo responderam os questionários aplicados no próprio local em que se encontravam e, com vistas a garantir o anonimato, estes deveriam colocá-los em uma urna lacrada. A urna somente foi aberta depois que todos os questionários foram coletados.

Foram incluídos no presente estudo, os indivíduos que, cumulativamente, atenderam aos seguintes critérios: (a) ser homem; (b) ter orientação sexual homossexual; (c) ter no mínimo 18 anos completos na data da coleta das informações e; (d) responder todo o questionário de forma anônima e assinar o Termo de Consentimento Livre e Esclarecido.

Para a caracterização dos participantes, foi utilizado um questionário autoaplicado, contendo informações acerca da orientação sexual, idade, altura, peso, idade da primeira relação sexual, grau de escolaridade e situação conjugal. A orientação de gênero (masculino, feminino e andrógeno) foi avaliada com base na escala existente na literatura, ${ }^{3}$ a qual o número 0 , significa exclusivamente masculino, e o 6 é utilizado para o indivíduo que percebe-se como exclusivamente feminino, tendo com um ponto mediano o número 3 (andrógeno).

$O$ peso e a altura foram obtidos através de medidas autorreferidas. A validade destas medidas tem sido comprovada com a população adulta brasileira. ${ }^{9}$ Para classificação do IMC utilizaram-se os pontos de corte estabelecido pela World Health Organization. É importante destacar que devido à pequena frequência de baixo peso (apenas dois participantes) e a ausência de obesidade na amostra estudada, optou-se pelo agrupamento de classes. Desta forma, o IMC da amostra foi dividido em "eutrófico", incluindo as categorias baixo peso e eutrófico; e "excesso de peso".
Para avaliar a insatisfação corporal dos homens homossexuais, utilizou-se a escala de desenhos de nove silhuetas,$^{10}$ a qual apresenta uma sequência de figuras de imagens corporais com nove variações em ordem crescente de tamanho corporal. O participante da pesquisa deveria apontar: 1) aquela figura que melhor o representava no momento atual e 2) apontar, posteriormente, a silhueta que representasse o seu ideal.

Feito isso, o nível de satisfação ou insatisfação com a imagem corporal foi obtida por meio da subtração da autopercepção da imagem corporal atual, pela autopercepção da imagem ideal. Os valores poderiam variar entre $-8 a+8$, os quais sejam: -8 a -1 (insatisfeito por magreza), 0 (satisfeito) e, por fim, 1 a 8 (insatisfeito por excesso de peso).

O nível de atividade física foi coletado por meio de uma questão, a qual o participante deveria responder: “Você pratica exercício físico regularmente?" Deveriam ser consideradas atividades físicas de intensidade moderada à vigorosa, no mínimo de 150 minutos por semana. Com isso, utilizaram-se as recomendações de estudos prévios: ativo 150 minutos ou mais de atividade física por semana; e insuficientemente ativo, menos de 150 minutos por semana. ${ }^{11}$

Os dados foram tabulados e analisados no programa estatístico Statistical Package for the Social Science (SPSS for Windows), versão 20.0, adotando um nível de significância de $\alpha<0,05$. Para caracterização da amostra foi empregada análise descritiva por meio dos valores de frequência absoluta e relativa. Os testes de Qui-quadrado e Exato de Fisher foram empregados para verificar a associação entre a orientação sexual e as variáveis independentes: grau de escolaridade, situação conjugal e orientação de gênero, IMC e atividade física.

Posteriormente, realizou-se a análise de Regressão Logística, estimando-se a razão de chances (odds ratio) e intervalos de confiança de $95 \%$, para verificar a associação entre a insatisfação com a imagem corporal e as variáveis independentes.

\section{RESULTADOS}

Neste estudo a média de idade dos participantes, homossexuais e bissexuais, foi de 26,49 anos (dp 6,20) com IMC de 23,96 (dp 2,79) e relataram que sua primeira relação sexual foi próximo aos 16 anos, conforme ilustra a tabela 1.

Tabela 1 - Média do somatório de seis dobras (S ADIP) em cada fase do ciclo menstrual.

\begin{tabular}{lcc}
\hline Variável & $\mathbf{x}$ & $\mathbf{D p}$ \\
Idade (em anos) & 26,49 & 6,20 \\
\hline IMC & 23,96 & 2,79 \\
Idade da primeira relação sexual & 15,89 & 1,65 \\
\hline
\end{tabular}

A tabela 2 ilustra que, entre os 646 participantes deste estudo, a maioria $(61,30 \%)$ dos homens deste estudo possui grau de escolaridade acima do ensino médio $(n=396), 608$ são solteiros $(94,12 \%), 450$ estão insatisfeitos com a imagem corporal $(69,70 \%)$, sendo 355 por excesso (55\%) e 95 por magreza $(14,70 \%)$, além de 607 perceberem-se com orientação de gênero masculino (96,96\%). Em relação ao IMC dos participantes, 230 estão na categoria de excesso de peso $(35,60 \%)$. Apenas 73 são insuficientemente ativos $(11,30 \%)$. 
Tabela 2 - Distribuição da amostra em relação à percepção da imagem corporal, orientação de gênero, IMC e nível de atividade física de homens homossexuais e bissexuais, Florianópolis, Santa Catarina, Brasil, 2015.

\begin{tabular}{lcc}
\hline Grau de escolaridade & $\mathbf{n}$ & $\%$ \\
\hline Ensino médio completo & 250 & 38,70 \\
Ensino superior & 396 & 61,30 \\
\hline Situação conjugal & & \\
Sem companheiro & 608 & 94,12 \\
Com companheiro & 38 & 5,88 \\
\hline Percepção da imagem corporal & & \\
Satisfeito & 196 & 30,30 \\
Insatisfeito por excesso & 355 & 55,00 \\
Insatisfeito por magreza & 95 & 14,70 \\
\hline Orientação de gênero & & \\
Masculino & 607 & 93,96 \\
Feminino & 39 & 6,04 \\
\hline IMC & & \\
Eutrófico & 416 & 64,40 \\
Excesso de peso & 230 & 35,60 \\
\hline Atividade Física & & \\
Insuficientemente ativo & 73 & 11,30 \\
Ativo & 573 & 88,70 \\
\hline Orientação sexual & 75 & 11,60 \\
Bissexual & 571 & 88,40 \\
Homossexual & 646 & 100 \\
\hline Total & & \\
\hline
\end{tabular}

Conforme a tabela 3, nota-se que os participantes homossexuais deste estudo, 551 consideram-se com orientação de gênero masculino (96,50\%), 375 apresentaram maior grau de escolaridade $(65,67 \%), 534$ vivem sem companheiro $(93,52 \%), 372$ foram classificados como eutróficos $(65,15 \%)$ e 383 estão insatisfeitos com a imagem corporal, sendo 288 insatisfeitos por excesso $(50,44 \%)$ e 95 insatisfeitos por magreza $(16,64 \%)$.

A proporção de homens homossexuais foi de $571(88,4 \%)$. Tanto na análise bruta, quanto na ajustada notou-se associação entre a orientação sexual e a
Tabela 3 - Orientação sexual homossexual, de acordo com as variáveis independentes em homens homossexuais, Florianópolis, Santa Catarina, Brasil. 2015.

\begin{tabular}{|c|c|c|c|c|c|}
\hline \multirow{2}{*}{$\begin{array}{l}\text { Variável } \\
\text { Orientação de gênero }\end{array}$} & \multicolumn{2}{|c|}{ Total } & \multicolumn{2}{|c|}{ Homossexual } & \multirow[b]{2}{*}{$p$} \\
\hline & $\mathbf{n}$ & $\%$ & $\mathbf{n}$ & $\%$ & \\
\hline Masculino & 607 & 93,96 & 551 & 96,50 & $\leq 0,001$ \\
\hline Feminino & 39 & 6,04 & 20 & 3,50 & \\
\hline \multicolumn{6}{|l|}{ Grau de escolaridade } \\
\hline Ensino médio completo & 250 & 38,70 & 196 & 35,33 & $\leq 0,001$ \\
\hline Ensino superior & 396 & 61,30 & 375 & 65,67 & \\
\hline \multicolumn{6}{|l|}{ Situação conjugal } \\
\hline Sem companheiro & 608 & 94,12 & 534 & 93,52 & 0,075 \\
\hline Com companheiro & 38 & 5,88 & 37 & 6,48 & \\
\hline \multicolumn{6}{|l|}{ IMC } \\
\hline Eutrófico & 416 & 64,40 & 372 & 65,15 & 0,270 \\
\hline Excesso de peso & 230 & 35,60 & 199 & 34,85 & \\
\hline \multicolumn{6}{|l|}{ Imagem corporal } \\
\hline Satisfeito & 196 & 30,34 & 188 & 32,92 & \\
\hline Insatisfeito por excesso & 355 & 54,96 & 288 & 50,44 & $\leq 0,001$ \\
\hline Insatisfeito por magreza & 95 & 14,70 & 95 & 16,64 & \\
\hline \multicolumn{6}{|l|}{ Atividade física } \\
\hline Ativo & 573 & 88,70 & 525 & 91,94 & $\leq 0,001$ \\
\hline Insuficientemente ativo & 73 & 11,30 & 46 & 8,06 & \\
\hline
\end{tabular}

orientação de gênero $O R=340,24(48,51-2386,46)$, grau de escolaridade $O R=0,20(0,003-0,12)$ e a insatisfação com a imagem corporal por excesso $O R=0,124$ $(0,49-0,314)$.

\section{DISCUSSÃO}

A proporção de homens homossexuais foi de 571 $(88,4 \%)$. Os resultados deste estudo apontaram a maioria dos homossexuais com orientação de gênero masculino $(96,50 \%)$ e apenas 20 indivíduos percebem-se como feminino $(3,50 \%)$. Corroborando com os achados desta pesquisa, ${ }^{12}$ encontra-se em atletas homossexuais americanos a autopercepção do gênero masculino em

Tabela 4 - Oddsratio e intervalos de confiança da análise bruta e ajustada da associação entre a orientação sexual e as variáveis sociodemográficas, índice de massa corporal (IMC) e percepção da imagem corporal em homens homossexuais, Florianópolis, Santa Catarina, Brasil, 2015.

\begin{tabular}{|c|c|c|c|c|c|}
\hline \multirow[b]{3}{*}{ Variável } & \multicolumn{5}{|c|}{ Homossexual } \\
\hline & \multicolumn{2}{|c|}{ Análise bruta } & \multirow[b]{2}{*}{ valor de $p$} & \multicolumn{2}{|c|}{ Análise ajustada } \\
\hline & OR & IC $95 \%$ & & OR & IC $95 \%$ \\
\hline \multicolumn{6}{|l|}{ Orientação de gênero } \\
\hline Masculino & 9,37 & $4,71-18,55$ & $<0,001$ & 340,24 & $48,51-2386,46$ \\
\hline Feminino & \multicolumn{2}{|c|}{1,0} & & \multicolumn{2}{|c|}{1,0} \\
\hline \multicolumn{6}{|l|}{ Situação conjugal } \\
\hline Com companheiro & 5,127 & $0,69-37,92$ & 0,109 & 0,287 & $0,23-3,57$ \\
\hline Sem companheiro & \multicolumn{2}{|c|}{1,0} & & \multicolumn{2}{|c|}{1,0} \\
\hline \multicolumn{6}{|l|}{ Grau de escolaridade } \\
\hline Ensino médio completo & 0,20 & $0,119-0,35$ & $<0,001$ & 0,20 & $0,003-0,12$ \\
\hline Superior completo & \multicolumn{2}{|c|}{1,0} & & \multicolumn{2}{|c|}{1,0} \\
\hline \multicolumn{6}{|l|}{ IMIC } \\
\hline Eutrófico & 1,31 & $0,81-2,15$ & 0,271 & 1,00 & $0,567-1,79$ \\
\hline Excesso de peso & \multicolumn{2}{|c|}{1,0} & & \multicolumn{2}{|c|}{1,0} \\
\hline \multicolumn{6}{|l|}{ Imagem corporal } \\
\hline Insatisfação por magreza & 68743610,3 & $0-0$ & 0,997 & 13329,92 & $0-0$ \\
\hline Insatisfeito por excesso & 0,183 & $0,86-0,389$ & $<0,001$ & 0,124 & $0,49-0,314$ \\
\hline Satisfeito & \multicolumn{2}{|c|}{1,0} & & \multicolumn{2}{|c|}{1,0} \\
\hline
\end{tabular}

$O R=$ oddsratio (razão de chance). IC = intervalo de confiança. Teste de Wald. Categoria de referência (bissexual). Valores em negrito foram significativos. 
todos os participantes $(100,00 \%)$ e em outro estudo, ${ }^{13}$ em seu estudo, $89,30 \%$ dos participantes se autodeclararam como masculino. De modo contrário, autores ${ }^{14}$ encontraram semelhanças em homossexuais que se autopercebem como masculino $(54,40 \%)$ e feminino $(45,60 \%)$, e também, em estudo similar ${ }^{(15)}$ encontrou em adolescentes, que se autodeclararam homossexual, bissexual e heterossexual, a orientação de gênero masculino, respectivamente $(48,75 \% ; 53,64 \%$ e $68,75 \%)$.

Tais informações nos permitem compreender que a orientação de gênero masculino pode ou não estar direcionada aos indivíduos homossexuais.

Os homossexuais tiveram maior escolaridade $(65,67 \%)$, em relação aos bissexuais participantes deste estudo. Pesquisa ${ }^{16}$ aponta que os homossexuais tendem a permanecer mais na escola em relação aos heterossexuais, sendo que alguns fatores foram encontrados, entre eles: não terem filhos precocemente $(89,54 \%)$, considerar que devem superar as barreiras advindas do preconceito social $(76,42 \%)$ e considerar que os estudos são a porta de entrada para uma sociedade mais igualitária $(53,44 \%)$.

Contrariamente e esse achado, pesquisa ${ }^{16}$ realizada com 748 homossexuais nos Estados Unidos, encontrou alta evasão escolar nesta população (64,7\%). E ainda, estudo ${ }^{17}$ aponta que os jovens bissexuais frequentam por mais tempo a escola pelo fato da sua orientação sexual ser muitas vezes velada e, com isso, sofrerem menos preconceitos e discriminação entre os colegas de turma.

Embora 573 (88,70\%) participantes tenham sido classificados como fisicamente ativos, a maioria $(n=383)$ estão insatisfeitos com a imagem corporal, tanto por excesso $(n=288 ; 50,44 \%)$ quanto magreza $(n=95 ; 16,64 \%)$. De acordo com pesquisa já realizada ${ }^{18}$ a prática de atividade física regular pode ser considerada uma estratégia para intervir na satisfação/insatisfação corporal, haja vista que propicia modificações na composição corporal, aprimoramento da forma e simetria do corpo. Embora a mídia e a sociedade são influenciadores na construção da figura do corpo ideal. ${ }^{19}$

Uma das razões dos homens homossexuais estarem insatisfeitos com a imagem corporal e darem importância ao tamanho do músculo são a sua associação com a masculinidade e status social. ${ }^{20}$ Segundo o estudo a insatisfação com o corpo, encontrada em $74,32 \%$ de bissexuais e homossexuais pode ser gerada a partir da crença de que na sociedade os outros homens preferem corpos mais fortes por remeter a maior masculinidade e autoconfiança.

Mais recentemente, estudo ${ }^{21}$ trouxe evidência que a insatisfação corporal pode ser um fator de risco maior para homossexuais com transtornos alimentares, pois $86,56 \%$ dos gays sofrem ou já sofreram de anorexia ou bulimia.

Um estudo realizado ${ }^{22}$ com o objetivo de comparar a insatisfação corporal entre 134 homens homossexuais e 119 heterossexuais na Austrália, verificou que, tanto os homens homossexuais e heterossexuais desejaram, igualmente, ser mais magro $(68,5 \%)$ e mais musculoso $(82,9 \%)$. No entanto, a insatisfação com o corpo nessa pesquisa foi maior para os homens gays $(67,08 \%)$. Assim, tal fato indica que o cuidado e a preocupação com o corpo é predominante em todas as pessoas com orientação sexual diferente.

Predominantemente, os participantes homossexu- ais deste estudo foram classificados como ativos fisicamente $(91,94 \%)$, eutrófico $(65,15 \%)$ e insatisfeitos com a imagem corporal por excesso $(50,44 \%)$. Percebendo-se assim que, embora fisicamente ativos e com peso dentro dos padrões da normalidade, encontram-se insatisfeitos com a imagem corporal. De modo contrário a esses achados, estudo evidencia ${ }^{24}$ que indivíduos gays insatisfeitos com a representação da imagem corporal tendem a não realizarem atividade física regularmente.

Estudo $^{24}$ têm demonstrado evidências da associação da imagem corporal com IMC, todavia, a associação com a prática de atividade física é mais recente e encontra-se em processo de construção.

Não houve diferença estatística significativa entre homossexuais e bissexuais na variável situação conjugal $(p=0,075)$ e IMC $(p=0,270)$, demonstrando que os homossexuais não diferem de outros homens com orientação sexual bissexual. No entanto, autores ${ }^{25}$ encontraram que homens gays tem índice de IMC menor do que heterossexuais

Tanto na análise bruta, quanto na ajustada notou-se associação entre a orientação sexual e a orientação de gênero $O R=340,24(48,51-2386,46)$, grau de escolaridade $O R=0,20(0,003-0,12)$ e a insatisfação com a imagem corporal por excesso $\mathrm{OR}=0,124$ $(0,49$ - 0,314). Demonstrando que os participantes com orientação sexual homossexual têm mais chances de terem mais escolaridade e mais insatisfação corporal por excesso em relação aos bissexuais.

Estudo $^{26}$ realizado com 7.376 jovens americanos que os gays e bissexuais tem $65,32 \%$ mais chances e entre eles, $47,43 \%$ dos homossexuais já tiveram dificuldades escolares e $71,52 \%$ pensaram ou pensam em evadir-se da escola antes do concluir o ensino médio, entre os motivos encontrados, destaca-se, ter sido vítima de bullyng $(32,73 \%)$, ser vítima de violência homofóbica $(30,54 \%)$, uso de drogas $(5,32 \%)$ e sentimentos de depressão e tentativas suicidas (2,93\%). Assim, embora os achados na presente pesquisa tenham apontado alto grau de escolaridade entre os homossexuais, esses resultados divergem da literatura encontrada e, com isso, aponta um panorama indicativo que é preciso que escolas tenham a capacidade de identificar e desconstruir os resultados negativos que gays e bissexuais podem encontrar durante sua vida escolar.

\section{CONSIDERACÕES FINAIS}

Os resultados deste estudo evidenciam que alguns dos resultados desta pesquisa foram similares e outros contrários aos encontrados na literatura nacional e internacional.

A proporção de homens, com orientação sexual homossexual, foi de $88,4 \%$, sendo que a maioria deles são fisicamente ativos, foram classificados como eutróficos, embora estão insatisfeitos com a imagem corporal. E ainda, eles tem mais chances de serem masculinos e estarem insatisfeitos por excesso do que os homens bissexuais, participantes deste estudo.

Nesse sentido, sugere-se que sejam realizadas novas investigações a fim de que os resultados encontrados possam ser comparados com outros estudos realizados em homossexuais, bissexuais e heterossexuais de diferentes regiões do país. 


\section{REFERÊNCIAS}

1. Cardoso FL. The relationship between sexual orientation and gender identification among males in a cross-cultural analysis in Brazil, Turkey and Thailand. Sexuality \& Culture 2013;17(4):568-97. doi: 10.1007/s12119-012-9161-2

2. Cardoso LRD, Malbergier A, Figueiredo TFB. O consumo de álcool como fator de risco para a transmissão das DSTs/HIV/ Aids. Rev. psiquiatr. clín 2008;35(Suppl 1):70-5.

3. Kinsey, A.; Pomeroy, W.; Martin, C. Sexual Behavior in the Human Male. Saunders, Philadelphia, 1948.

4. Ghorayeb DB, Saúde mental, qualidade de vida, religiosidade e identidade psicossocial nas homossexualidades. Dissertação de Mestrado [Unicamp], Campinas, SP: 2012.

5. Carper TL, Negy C, Tantleff-Dunn S. Relations among media influence, body image, eating concerns, and sexual orientation in men: a preliminary investigation. Body Image 2010;7(4):301-9. doi: 10.1016/j.bodyim.2010.07.002

6. Pinto VM, Tancredi MV, Alencar HDR De, Camolesi E, Holcman MM, Grecco JP, Grangeiro A, Grecco ETO. Prevalência de Sífilis e fatores associados a população em situação de rua de São Paulo, Brasil, com utilização de Teste Rápido. Rev Bras Epidemiol 2014;17(2):341-54. doi: 10.1590/1809-4503201400020005ENG

7. Guimarães, CD. O homossexual visto por entendidos. Rio de Janeiro: Garamond, 2004.

8. Brasil. Ministério do Turismo. 2016. O turismo como destino LGBT. Disponível em: <http://www.turismo.gov.br/turismo/ noticias/todas _noticias/20110523-2.html>. 2016. Acesso em: 11 jun. 2016.

9. Coqueiros RS, Borges LJ, Araújo VC, Pelegrini A, Barbosa AR. Medidas autorreferidas são validas para avaliação do estado nutricional brasileira? Rev Bras Cineantropom Desempenho Hum 2009;11(1):113-19.

10. Stunkard AJ, Sørensen T, Schulsinger F. Use of the Danish Adoption Register for the study of obesity and thinness. The Genetics of Neurological and Psychiatric Disorders 1983; 60:115-20.

11. Hallal PC, Andersen LB, Bull FC, Guthold R, Haskell W, Ekelund $U$. Global physical activity levels: surveillance progress, pitfalls, and prospects. The Lancet 2012; 380(9838):247-57. doi: 10.1016/S0140-6736(12)60646-1

12. Filiault SM, Drummond MJN. Gay atheletes perceptions of body hair. JMS 2013;21(2):206-13.

13. Kane GD. Revisiting gay men's body image issues: exposing the fault lines. Rev Gen Psychol 2010;14(4):311-17. doi: 10.1037/a0020982

14. Brannan DJ, Asakura K, George C, Newman PA, Giwa
S, Hart TA, Souleymanov R, Betancourt G. Never reflected anywhere: Body image among ethnoracialized gay and bisexual men. Body Image 2012;10(3):389-98. doi: 10.1016/j.bodyim.2013.03.006

15. Saewyc EM. Research on adolescence, sexual orientation, health disparities, stigma and resilience. J Reas Adolesc 2011;21(1):256-72. doi: 10.1111/j.1532-7795.2010.00727.x

16. Hammack PL, Thompson EM, Pilecki A. Configurations of identity among sexual minority youths: context, desire, and narrative. J Youth Adolesc 2009;38(7):867-83.

17. Berlan ED, Corliss HL, Field AE, Goodman E, Austin SB. Sexual Orientationand Bullying Among Adolescents in the Growing Up Today Study. J Adolesc Health 2010;46(4):36671. doi: 10.1016/j.jadohealth.2009.10.015

18. Gonçalves CO, Campana AN, Tavares MC. Influência da atividade física na imagem corporal: uma revisão bibliográfica. Motri 2012;8(2):70-82. doi: 10.6063/ motricidade.8(2).716

19. Alves D, Pinto M, Alves S, Mota A, Leirós V. Cultura e imagem corporal. Motricidade 2009;5(1):1-20.

20. Kimmel SB, Mahalik JR. Measuring masculine body ideal distress: development of a measure. JMS 2010;3(1):1-10.

21. Smith AM, Patrick K, Heywood W, Pitts MK, Richters J, Shelley JM, Simpson JM, Ryall R. Body mass index, sexual difficulties and sexual satisfaction among people in regular heterosexual relationships: a population-based study. Internal Medicine Journal 2012;42(6):641-51. doi: 10.1111/j.14455994.2011.02597.x

22. Tiggemann $M$, Martins $Y$, Kirkbride $A$. Oh to be lean and muscular: Body image ideals in gay and heterosexual men. Psychology of Men \& Masculinity 2007;8(1):15-24. doi: 10.1037/1524-9220.8.1.15

23. Anton SD, Perri MG, Riley JR. Discrepancy between actual and ideal images: impact on eating and exercise behaviors. Eat Behav 2010;1(2):153-60.

24. Silva D, Nunes E. Imagem corporal e estágios de mudança de comportamento para atividade física em universitários. Rev Bras Ativ Fis e Saúde 2014;19(5):597-600. doi: 10.12820/ RBAFS.V.19N5P597

25. Lavender JM, De Young KP, Anderson DA. Eating Disorder Examination Questionnaire (EDE-Q): norms for undergraduate men. Eat Behav 2010;11(2):119-21. doi: 10.1016/j.eatbeh.2009.09.005

26. Birkitt M, Espelage DL, Koenig B. LGB and questioning students in schools: The moderating effects of homophobic bullying and school climate on negative outcomes. J Youth and Adolescence 2010;38(7):989-1000. doi: 10.1007/ s10964-008-9389-1

Como citar: TEIXEIRA, Fabiano Augusto; CARDOSO, Fernando Luiz. Orientação sexual e fatores associados em homens homossexuais. Cinergis, Santa Cruz do Sul, v. 18, n. 2, mar. 2017. ISSN 2177-4005. Disponível em: <https://online.unisc.br/ seer/index.php/cinergis/article/view/8301 >. Acesso em: 29 mar. 2017. doi:http://dx.doi.org/10.17058/cinergis.v18i2.8301. 